\title{
ON THE LOCAL TRANSFORMED BASED METHOD FOR PARTIAL INTEGRO-DIFFERENTIAL EQUATIONS OF FRACTIONAL ORDER
}

\author{
MARJAN UDDIN AND MUHAMMAD TAUFIQ
}

Received 13 November, 2019

\begin{abstract}
In the present work, a localized Laplace transform based numerical scheme is constructed for fractional partial integro-differential equations with weakly singular kernels. By application of the Laplace transform the time variable is eliminated and the spatial derivatives are approximated by RBF which transformed the problem into a linear system of equations. The resultant system lead to differentiation matrices which are sparse contrary to large global collocation matrices. The inverse Laplace transform is then computed numerically using contour integration to recover the solution. The efficiency and accuracy of the method is demonstrated by several numerical experiments. The results obtained by the present method are compared with analytical solutions and other numerical methods.
\end{abstract}

2010 Mathematics Subject Classification: 65R10; 65R20

Keywords: local method, Laplace transform, fractional order partial integro-differential equations, weakly singular kernels, RBF

\section{INTRODUCTION}

In this work, we consider the following type of fractional order partial integrodifferential equations with memory term of convolution type

$$
D_{t}^{\alpha} v(x, t)=\gamma v_{x x}(x, t)+\int_{0}^{t} v_{x x}(x, z) \beta(t-z) d z+f(x, t),
$$

where $0<\alpha<1,0 \leq t \leq T, f(x, t)$ is given function and $\beta(t)=t^{-\frac{1}{2}}$ is the weakly singular kernel, with the following initial condition

$$
v(x, 0)=v_{0}, x \in \Omega,
$$

and with the RBC boundary condition

$$
\mathcal{B} v(x, t)=0, x \in \partial \Omega \text {. }
$$


In recent years, researchers have shown growing interest in the study of fractional integro-differential equations. In the last decades such type of equations have numerous applications in the fields of science and engineering like as finance, hydrology, physics and control theory, cosmology, solid mechanics, chemistry, economics, bioengineering, statistical mechanics, viscoelastic materials, and control theory $[1,11,29,30]$. Fractional order integro-differential equations successfully modeled many physical phenomenon. The analytical solution of these equations are not possible or are hard to find. Therefore researchers have solved these problems numerically. For the solution of fractional integro-differential equations (FIDEs) various numerical method have been used over the years, some of these may be found in the references [9, 25, 26, 32, 33].

Brunner and co-authors have developed very efficient collocation methods for the solution of Volterra integral equations, some of these can be found in the references $[2,3,7]$, and some other accurate numerical methods are referred in $[8,27,31,42]$. Several mathematical models and simulations of real world problems usually expressed in terms of functional equations. These models include PDEs and FIDEs. However, some practical problems such as modeling and simulations in science and engineering can be formulated in terms of fractional order partial integro-differential equations. These equations are often used to model heat conduction in material with memory term, chemical, nuclear reactor design and fluid dynamics $[4,6,12,15,17-$ $19,23,35,36,38,40]$.

Analytical solution of many integral equations are hard to find or either do not exist so we need an efficient and accurate numerical method. Many numerical methods have been used over the last ten decades, including finite element methods [4, 13], finite difference methods [35] and Laplace transform methods [39], Spectral collocation method and Galerkin methods [12, 14,41] for approximating integrals and integral equations. The authors in $[16,21]$ have been considered numerical methods for partial integro-differential equations.

Recently applications of radial basis functions (RBFs) is an active area of research. These RBF based meshfree techniques are employed for finding approximate solution of partial differential equations (PDEs) in irregular shaped domains. The detail of such methods can be found [10]. The researchers have shown interest in derivations and implementations of such methods for solving the various types of problems. The mesh-based methods such as the finite difference and finite element methods are not well suited for problems in complex geometry. In the present work, a new technique Laplace transform based local radial basis functions is applied for solving problems $(1.1)-(1.3)$ numerically. By application of Laplace transform the time variable is eliminated and the resulting equation is solved by local radial basis functions method. For getting high accuracy some contours like parabolic and hyperbolic are also used in the numerical inversion of the Laplace transform. 


\section{PRELIMINARIES}

In this section, we provide some definitions and notations of the fractional calculus [22,28].

Definition 1. The Caputo derivative of fractional order is defined by

$$
D_{t}^{\alpha} v(t)=\frac{1}{\Gamma(n-\alpha)} \int_{0}^{t} \frac{1}{(t-z)^{\alpha+1-n}} \frac{d^{n}}{d s^{n}} v(z) d z
$$

where, $n-1<\alpha<n \in z^{+}$.

Definition 2. The Laplace transform of a given function $v(t)$, where $t \geq 0$ is defined as

$$
\hat{v}(z)=\mathcal{L}\{v(t)\}=\int_{0}^{\infty} e^{-z t} v(t) d t,
$$

if this improper integral converges.

Lemma 1. Assume the function $v(t)$ is continuous over $0 \leq t \leq t_{n}$, if there are some constants $K_{1}, K_{2}$, with the property,

$$
\left|e^{-K_{2} t} v(t)\right|<K_{1}, \forall t>t_{n}
$$

then the Laplace transform of $v(t)$ exists.

Lemma 2. If $v(t) \in C^{p}[0, \infty)$, with $\alpha \in(n-1, n) \in z^{+}$, then the Laplace transform of the Caputo fractional derivative is defined as

$$
\mathcal{L}\left\{D_{t}^{\alpha} v(t)\right\}(z)=z^{\alpha} \hat{v}-\sum_{i=0}^{n-1} z^{\alpha-i-1} v^{(i)}(0) .
$$

Theorem 1 (Lerch's theorem in [5]). If

$$
\hat{v}(z)=\int_{0}^{\infty} e^{-z t} v(t) d t, z>\gamma
$$

is satisfied by a continuous function $v(t)$, there is no other continuous function which satisfies the equation (2.5).

Theorem 2 (The Bromwich Inversion Theorem in [5]). Let $v(t)$ have a continuous derivative and let $|v(t)|<K e^{\gamma t}$, where $K$ and $\gamma$ are positive constants. Define $\hat{v}(z)=$ $\int_{0}^{\infty} e^{-z t} v(t) d t, \operatorname{Re}(z)>\gamma$, then

$$
v(t)=\frac{1}{2 \pi i} \int_{\xi-i \infty}^{\xi+i \infty} \hat{v}(z) e^{z t} d z .
$$




\section{Description of the Method}

\subsection{Time discretization via Laplace transform}

Now the Laplace transform is applied to eliminate the time variable and the resultant equations are solved using local meshless method. Application of Laplace transform to problem (1.1) - (1.3) gives

$$
\begin{gathered}
z^{\alpha} \hat{v}(x, z)-z^{\alpha-1} v_{0}=\hat{\beta}(z) \mathcal{D} \hat{v}_{x x}(x, z)+\hat{f}(x, z), x \in \Omega \subset \mathbb{R}^{d}, \\
\mathcal{B} \hat{v}(x, z)=\hat{h}(z), x \in \partial \Omega .
\end{gathered}
$$

The following system of equations is obtained

$$
\begin{gathered}
\left(z^{\alpha} I-\hat{\beta}(z) \mathcal{D}\right) \hat{v}(x, z)=z^{\alpha-1} v_{0}+\hat{f}(x, z), x \in \Omega \subset \mathbb{R}^{d}, \\
\mathcal{B} \hat{v}(x, z)=\hat{h}(z), x \in \partial \Omega .
\end{gathered}
$$

The solution $\hat{v}(x, z)$ of the transformed problem (3.3)-(3.4) will be approximated using local RBF method. Consequently by using numerical inversion we get the original solution $v(x, t)$ of the given problem $(1.1)-(1.3)$.

\subsection{Spatial discretization via radial kernels}

In this section we need to discretize the transform problem (3.3)-(3.4) for the linear operators $\mathcal{B}$ and $\mathcal{L}$ by using local radial basis functions [37]. Consider the centers $\left\{x_{1}, \ldots, x_{m}\right\} \subset \Omega \subset \mathbb{R}^{d}, d \geq 1$, where $\Omega$ is bounded domain. To approximate the unknown function $\hat{v}(x)$ at the functional values $\left\{\hat{v}\left(x_{j}\right), j=1,2, \ldots, m\right\}$, the local interpolant at each $x_{j} \in \Omega$ takes the form

$$
\hat{v}\left(x_{j}\right)=\sum_{x_{k} \in \Omega_{j}} \sigma_{k}^{j} \varphi^{j}\left(\left\|x_{j}-x_{k}\right\|\right),
$$

where, $\sigma^{j}=\left[\sigma_{1}^{j}, \sigma_{2}^{j}, \ldots, \sigma_{n}^{j}\right]$ is vector of unknown coefficients, and $r_{j k}=\left\|x_{j}-x_{k}\right\|$ is the norm between nodes $x_{j}$ and $x_{k}, \varphi(r), r \geq 0$ is a radial kernel (Multi-quadric radial basis function) and $\Omega_{j} \subset \Omega$ is a local domain for around each $x_{j}$, contains $n$ neighboring nodes around the node $x_{j}$. So we have $m$ small size linear systems each of order $n \times n$ given by

$$
\left(\begin{array}{c}
\hat{v}_{1}^{j} \\
\hat{v}_{2}^{j} \\
\vdots \\
\hat{v}_{n}^{j}
\end{array}\right)=\left(\begin{array}{cccc}
\varphi_{11}^{j} & \varphi_{12}^{j} & \cdots & \varphi_{1 n}^{j} \\
\varphi_{21}^{j} & \varphi_{22}^{j} & \cdots & \varphi_{2 n}^{j} \\
\vdots & \vdots & \ddots & \vdots \\
\varphi_{n 1}^{j} & \varphi_{n 2}^{j} & \cdots & \varphi_{n n}^{j}
\end{array}\right)\left(\begin{array}{c}
\sigma_{1}^{j} \\
\sigma_{2}^{j} \\
\vdots \\
\sigma_{n}^{j}
\end{array}\right), j=1,2, \ldots, m,
$$

which can be denoted by

$$
\hat{\mathbf{V}}^{j}=\mathbf{P}^{j} \boldsymbol{\sigma}^{j}, j=1,2, \ldots, m
$$


where $\varphi_{l k}^{j}=\varphi^{j}\left(\left\|x_{l}-x_{k}\right\|\right), x_{l}, x_{k} \in \Omega_{j}$, the matrix $\mathbf{P}^{j}$ is called system matrix.

Next applying the operator $\mathcal{L}$ to (3.5), we get

$$
\mathcal{L} \hat{v}\left(x_{j}\right)=\sum_{x_{k} \in \Omega_{j}} \sigma_{k}^{j} \mathcal{L} \varphi^{j}\left(\left\|x_{j}-x_{k}\right\|\right)
$$

In vector form this gives,

$$
\mathcal{L} \hat{v}\left(x_{j}\right)=\mathbf{W}^{j} \cdot \sigma^{j}
$$

where $\mathbf{W}^{j}$ is given by

$$
\mathbf{W}^{j}=\mathcal{L} \varphi^{j}\left(\left\|x_{j}-x_{k}\right\|\right), x_{j}, x_{k} \in \Omega_{j},
$$

the unknown coefficients can be eliminated from equation (3.6)

$$
\sigma^{j}=\left(\mathbf{P}^{j}\right)^{-1} \hat{\mathbf{V}}^{j},
$$

by inserting the values of $\sigma^{j}$ in (3.8) we have,

$$
\mathcal{L} \hat{v}\left(x_{j}\right)=\mathbf{W}^{j}\left(\mathbf{P}^{j}\right)^{-1} \hat{\mathbf{V}}^{j}=\mathbf{N}^{j} \hat{\mathbf{V}}^{j}
$$

where,

$$
\mathbf{N}^{j}=\mathbf{W}^{j}\left(\mathbf{P}^{j}\right)^{-1}
$$

Hence the localized discretized form of the linear operator at each center $x_{j}$ gives

$$
\mathcal{L} \hat{v}=\mathbf{H} \hat{\mathbf{v}},
$$

where $\mathbf{H}$ is the sparse differentiation matrix of order $m \times m$ with $n$ non-zeros values while $m-n$ zeros values in each row, and $n$ denote the nodes in local sub-domain $\Omega_{j}$ for each $j$.

\section{NUMERICAL INVERSION TECHNIQUE}

In this section, the solution $v(x, t)$ of the problem (1.1) - (1.3) is obtained using the following Laplace inversion form

$$
v(x, t)=\frac{1}{2 \pi \mathrm{l}} \int_{\xi-1 \infty}^{\xi+1 \infty} \hat{v}(x, z) e^{z t} d z=\frac{1}{2 \pi \mathrm{l}} \int_{\Upsilon} e^{z t} \hat{v}(x, z) d z, \xi>\xi_{0},
$$

where $\Upsilon$ is suitable path joining $\xi-1 \infty$ to $\xi+1 \infty$. The solution of problem (1.1) $-(1.3)$ is obtained from the complex contour integral (4.1) which is called the Bromwich integral along the chosen path. The hyperbolic and parabolic contours are used in the present work [20,40]. The hyperbolic path [20] is given by

$$
z(\eta)=\omega+\lambda(1-\sin (\sigma-\imath \eta)), \text { for } \eta \in \mathbb{R},
$$

with $\lambda>0, \omega \geq 0,0<\sigma<\beta-\frac{1}{2} \pi$, and $\frac{1}{2} \pi<\beta<\pi$ (for detail see [20]). The optimal parameters corresponding to the hyperbolic path are given as $l(x)=\max (1, \log (1 / x))$, $r>0, \quad \mu=\bar{r}(1-\theta) / b, \quad \bar{r}=2 \pi r, \quad 0<\theta<1, \quad \rho_{r}=\theta \bar{r} \tau \sin (\sigma-r) / b, \quad b=$ $\cosh ^{-1}(1 /(\theta \tau \sin (\sigma))), \tau=t_{0} / T, 0<t_{0}<T, t_{0} \leq t \leq T, \lambda=\theta \bar{r} M /(b T), k=b / M$ 
and error bound is $\left(l\left(\rho_{r} M\right)\right)$ (see Theorem 3.1, [20]). Using the paths hyperbolic path defined the integral (4.1) becomes

$$
v(x, t)=\frac{1}{2 \pi \mathrm{l}} \int_{-\infty}^{\infty} e^{z(\eta) t} \hat{v}(x, z(\eta)) \dot{z}(\eta) d \eta,
$$

and equal width trapezoidal rule is used for approximation of (4.3) in the following equation

$$
v_{k}(x, t)=\frac{k}{2 \pi \mathrm{l}} \sum_{j=-M}^{M} \hat{v}\left(x, z_{j}\right) e^{z_{j} t} z_{j}, z_{j}=z\left(\eta_{j}\right), \eta_{j}=j k,
$$

where $k$ is step size.

\section{STABILITY}

In this section, we discuss stability of the system (3.3)-(3.4), and can be expressed as

$$
A \hat{\mathbf{v}}=\mathbf{b},
$$

where the matrix $A$ is known as the differentiation matrix of order $N \times N$ obtained in using local RBF method. From system (5.1) the stability constant is given by

$$
C=\sup _{\hat{\mathbf{v}} \neq 0} \frac{\|\hat{\mathbf{v}}\|}{\|A \hat{\mathbf{v}}\|},
$$

where the constant $C$ is finite which can be obtained by using some discrete norms $\|$.$\| on R^{N}$. Equation (5.2) gives

$$
\|A\|^{-1} \leq \frac{\|\hat{\mathbf{v}}\|}{\|A \hat{\mathbf{v}}\|} \leq C,
$$

Next in case of pseudoinverse $A^{\dagger}$ of $A$

$$
\left\|A^{\dagger}\right\|=\sup _{w \neq 0} \frac{\left\|A^{\dagger} w\right\|}{\|w\|} .
$$

Now we have

$$
\left\|A^{\dagger}\right\| \geq \sup _{w=A \hat{\mathbf{v}} \neq 0} \frac{\left\|A^{\dagger} A \hat{\mathbf{v}}\right\|}{\|A \hat{\mathbf{v}}\|}=\sup _{\hat{\mathbf{v}} \neq 0} \frac{\|\hat{\mathbf{v}}\|}{\|A \hat{\mathbf{v}}\|}=C .
$$

Hence equations (5.3) and (5.5) assures the boundedness of the stability constant $C$. To ensure numerical stability in solving system (5.1), Pseudoinverse can be used, but calculation may be computationally expansive. In case of square systems, we use the MATLAB built in function condest estimates

$$
C=\frac{\text { condest }\left(A^{\prime}\right)}{\|A\|_{\infty}}
$$




\section{APPLICATION OF THE METHOD}

In this section, we applied our numerical technique which is the combination of Laplace transform and localized RBF based meshfree method to approximate the time fractional partial integro-differential equations. The accuracy of the method is shown in the form of tables and graphs.

Example 1. Here we consider the model defined in equations (1.1) - (1.3) as our first test problem with the following function and boundary conditions

$$
\begin{gathered}
f(x, t)=\frac{1}{\Gamma(3-\alpha)}\left(x^{2}-1\right) t^{2-\alpha}-2 t^{2}-\frac{32}{15} t^{\frac{5}{2}}, \\
v(x, 0)=0, x \in(-1,1), v(0, t)=v(1, t)=0,0<t \leq T,
\end{gathered}
$$

where the actual solution [24] of above problem is $v(x, t)=t^{2}\left(x^{2}-1\right)$. The contour mentioned in (4.2) and the following optimal values of the parameter are used in the solution of the above problem.

$$
\begin{gathered}
\omega=2, \sigma=0.3812, \lambda=\frac{\theta r_{b} M}{b T}, x_{k}=h k, \theta=0.1, r_{b}=2 \pi r, r=0.3431, \\
h=b / M, b=\cosh ^{-1}\left(\frac{1}{\theta \tau \sin (\sigma)}\right), \tau=t_{0} / T, t_{0}=0.1, t=1, T=5 .
\end{gathered}
$$

The $l_{\infty}$ errors at various quadrature points $M$, collocation points $m \in \Omega$, stencils points $n \in \Omega_{i}$ and fractional order $\alpha$, and the error estimate $\varepsilon(c, M)=\left(e^{\frac{-c M}{\log (M)}}\right), c=1$ of the proposed numerical scheme are shown in Table 1. The error estimate $\varepsilon(c, M)$ decreasing exponentially for $11 \leq M \leq 91$, while at $M=50$ and $4 \leq n \leq 15$ the error estimate remain unchanged are shown in Table 1 and Figure 1 respectively. The numerical solutions obtained by Laplace transform based local meshless method are better than the results obtained by the method in [24].

Example 2. In this example, we apply the proposed numerical method to solve model (1.1) - (1.3) as a second test problem in [24] with

$$
f(x, t)=\cos (\pi x)\left(\frac{2}{\Gamma(3-\alpha)} t^{2-\alpha}+t^{2}+\frac{16}{15} t^{\frac{5}{2}}\right),
$$

with the following initial and boundary conditions

$$
v(x, 0)=0, x \in(0, \pi), v(0, t)=v(1, t)=0,0<t \leq T .
$$

The exact solution of the above problem is $v(x, t)=t^{2} \cos (x)$. The same set of parameters are used for the solution of Example 2 as well. The $l_{\infty}$ error norm is steadily decreasing from top to bottom in each column for $10 \leq M \leq 60$ and similarly the error is decaying slowly from left to right in each row by increasing value of $\alpha$ and are shown in Table 2 and Figure 2 respectively. The results obtained by the present method are more accurate than the results in [24]. 
TABLE 1. Numerical results using Laplace transform based local RBF method corresponding to Example 1.

\begin{tabular}{cccccc}
\hline$(m, n=45,5)$ & $\alpha=0.2$ & $\alpha=0.4$ & $\alpha=0.6$ & $\alpha=0.8$ & \\
\hline $\mathrm{M}$ & $l_{\infty}$ & $l_{\infty}$ & $l_{\infty}$ & $l_{\infty}$ & $\varepsilon(c, M)$ \\
\hline 11 & $5.29 \mathrm{e}-006$ & $5.31 \mathrm{e}-006$ & $5.34 \mathrm{e}-006$ & $5.37 \mathrm{e}-006$ & 0.0102 \\
21 & $5.62 \mathrm{e}-006$ & $5.39 \mathrm{e}-006$ & $5.14 \mathrm{e}-006$ & $4.89 \mathrm{e}-006$ & 0.0010 \\
41 & $1.62 \mathrm{e}-005$ & $1.56 \mathrm{e}-005$ & $1.49 \mathrm{e}-005$ & $1.41 \mathrm{e}-005$ & $1.60 \mathrm{e}-005$ \\
51 & $2.73 \mathrm{e}-005$ & $2.63 \mathrm{e}-005$ & $2.52 \mathrm{e}-005$ & $2.40 \mathrm{e}-005$ & $2.32 \mathrm{e}-006$ \\
71 & $3.99 \mathrm{e}-005$ & $3.85 \mathrm{e}-005$ & $3.69 \mathrm{e}-005$ & $3.52 \mathrm{e}-005$ & $5.83 \mathrm{e}-008$ \\
91 & $5.61 \mathrm{e}-005$ & $5.41 \mathrm{e}-005$ & $5.20 \mathrm{e}-005$ & $4.97 \mathrm{e}-005$ & $1.73 \mathrm{e}-009$ \\
\hline$(M, m=50,45)$ & & & & & \\
\hline $\mathrm{n}$ & & & & & \\
\hline 4 & $5.41 \mathrm{e}-004$ & $5.24 \mathrm{e}-004$ & $5.05 \mathrm{e}-004$ & $4.84 \mathrm{e}-004$ & $2.81 \mathrm{e}-006$ \\
5 & $3.00 \mathrm{e}-005$ & $2.89 \mathrm{e}-005$ & $2.77 \mathrm{e}-005$ & $2.63 \mathrm{e}-005$ & $2.81 \mathrm{e}-006$ \\
7 & $8.38 \mathrm{e}-005$ & $8.13 \mathrm{e}-005$ & $7.85 \mathrm{e}-005$ & $7.55 \mathrm{e}-005$ & $2.81 \mathrm{e}-006$ \\
9 & $6.58 \mathrm{e}-005$ & $6.37 \mathrm{e}-005$ & $6.14 \mathrm{e}-005$ & $5.89 \mathrm{e}-005$ & $2.81 \mathrm{e}-006$ \\
11 & $1.51 \mathrm{e}-004$ & $1.46 \mathrm{e}-004$ & $1.41 \mathrm{e}-004$ & $1.35 \mathrm{e}-004$ & $2.81 \mathrm{e}-006$ \\
15 & $2.13 \mathrm{e}-004$ & $2.06 \mathrm{e}-004$ & $1.98 \mathrm{e}-004$ & $1.90 \mathrm{e}-004$ & $2.81 \mathrm{e}-006$ \\
\hline$m=100$ & $L_{\infty}=7.4715 e^{-5}$ & {$[24]$} & & & \\
\hline \multicolumn{7}{c}{}
\end{tabular}

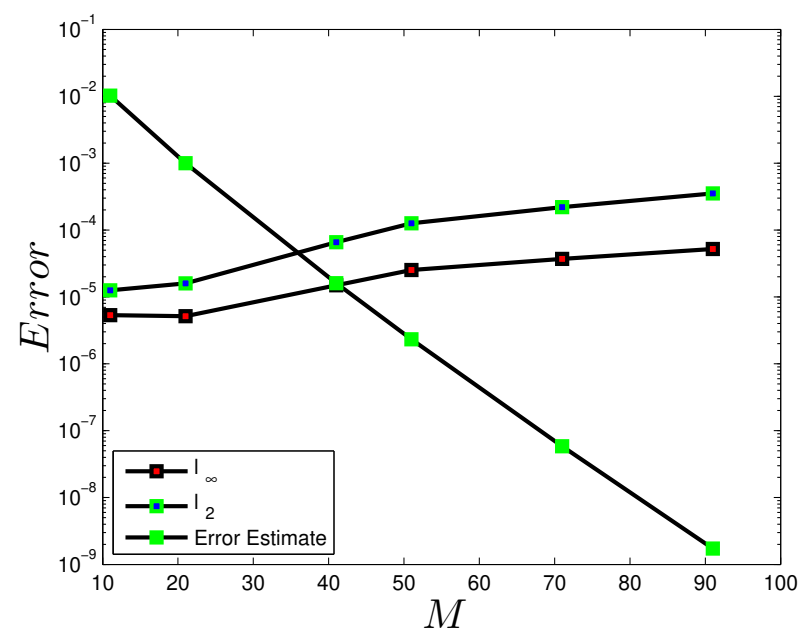

FIGURE 1. Approximate solution: error versus quadrature points corresponding to Example 1. 
TABLE 2. Numerical results of fractional partial-integro-differential equation at time $t=1 \in\left[t_{0}=0.1, T=5\right]$ by present method to Example 2.

\begin{tabular}{cccccc}
\hline$m=45, n=5$ & $\alpha=0.2$ & $\alpha=0.3$ & $\alpha=0.7$ & $\alpha=0.8$ & \\
\hline $\mathrm{M}$ & $l_{\infty}$ & $l_{\infty}$ & $l_{\infty}$ & $l_{\infty}$ & $\varepsilon(c, M)$ \\
\hline 10 & $1.18 \mathrm{e}-004$ & $1.17 \mathrm{e}-004$ & $1.14 \mathrm{e}-004$ & $1.13 \mathrm{e}-004$ & 0.0130 \\
20 & $1.33 \mathrm{e}-005$ & $1.33 \mathrm{e}-005$ & $1.31 \mathrm{e}-005$ & $1.31 \mathrm{e}-005$ & 0.0013 \\
30 & $8.10 \mathrm{e}-006$ & $8.10 \mathrm{e}-006$ & $8.09 \mathrm{e}-006$ & $8.09 \mathrm{e}-006$ & $1.47 \mathrm{e}-004$ \\
40 & $7.03 \mathrm{e}-006$ & $7.03 \mathrm{e}-006$ & $7.03 \mathrm{e}-006$ & $7.03 \mathrm{e}-006$ & $1.95 \mathrm{e}-005$ \\
50 & $6.58 \mathrm{e}-006$ & $6.58 \mathrm{e}-006$ & $6.58 \mathrm{e}-006$ & $6.58 \mathrm{e}-006$ & $2.81 \mathrm{e}-006$ \\
60 & $6.88 \mathrm{e}-006$ & $6.88 \mathrm{e}-006$ & $6.88 \mathrm{e}-006$ & $6.88 \mathrm{e}-006$ & $4.32 \mathrm{e}-007$ \\
\hline$M=50, n=5$ & \multicolumn{5}{c}{} \\
\hline $\mathrm{m}$ & $l_{\infty}$ & $l_{\infty}$ & $l_{\infty}$ & $l_{\infty}$ & $\varepsilon(c, M)$ \\
\hline 10 & 0.0068 & 0.0068 & 0.0068 & 0.0068 & 0.0130 \\
20 & $9.94 \mathrm{e}-005$ & $9.94 \mathrm{e}-005$ & $9.94 \mathrm{e}-005$ & $9.94 \mathrm{e}-005$ & 0.0013 \\
30 & $1.44 \mathrm{e}-004$ & $1.44 \mathrm{e}-004$ & $1.44 \mathrm{e}-004$ & $1.44 \mathrm{e}-004$ & $1.47 \mathrm{e}-004$ \\
40 & $3.44 \mathrm{e}-005$ & $3.44 \mathrm{e}-005$ & $3.44 \mathrm{e}-005$ & $3.44 \mathrm{e}-005$ & $1.95 \mathrm{e}-005$ \\
50 & $6.65 \mathrm{e}-006$ & $6.61 \mathrm{e}-006$ & $6.46 \mathrm{e}-006$ & $6.43 \mathrm{e}-006$ & $2.81 \mathrm{e}-006$ \\
60 & $4.22 \mathrm{e}-006$ & $4.18 \mathrm{e}-006$ & $3.99 \mathrm{e}-006$ & $3.95 \mathrm{e}-006$ & $4.32 \mathrm{e}-007$ \\
\hline \multicolumn{7}{c}{}
\end{tabular}

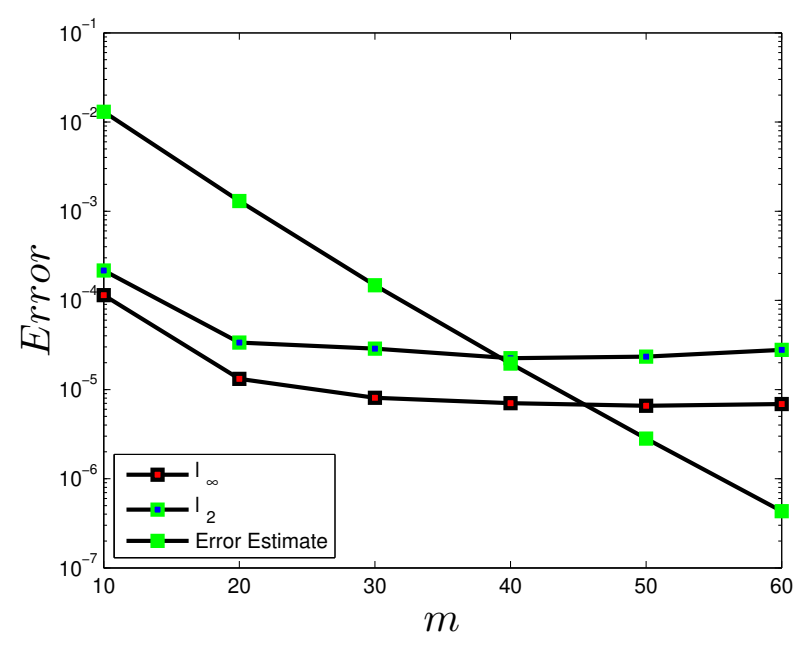

FIgURE 2. Approximate solution: error versus quadrature points corresponding to Example 2 
Example 3. In the third test problem, we apply the proposed method for model problem given in equations $(1.1)-(1.3)$, subject to the initial and boundary conditions and function $f$

$$
\begin{gathered}
f(x, t)=\sin (\pi x)\left[\frac{1}{\Gamma(2-\alpha)} t^{1-\alpha}+\pi^{2} t+\frac{4}{3} \pi^{2} t^{\frac{3}{2}}\right], \\
v(x, 0)=0, x \in(0,1], v(0, t)=v(1, t)=0,0<t \leq T,
\end{gathered}
$$

where the exact solution $v(x, t)=t \sin (\pi x)$ is taken for the purpose of comparison [24]. Various quadrature points $M$ along the hyperbolic $\Gamma$ contour (4.2) are used in approximating the solution. The $l_{\infty}$ errors at various $m \in \Omega$, stencils points $n$ in $\Omega_{i}$ and $\alpha$ are shown in Table 3 and Figure 3. From Table 4, we observed that the $l_{\infty}$ error is decaying algebraically for $20 \leq M \leq 60$ quadrature points. Hence the present method is highly convergent.

TABLE 3. Numerical results using Laplace transform method corresponding to Example 3.

\begin{tabular}{ccccc}
\hline$M=70, n=5$ & $\alpha=0.1$ & $\alpha=0.25$ & $\alpha=0.6$ & $\alpha=0.75$ \\
\hline $\mathrm{N}$ & $l_{\infty}$ & $l_{\infty}$ & $l_{\infty}$ & $l_{\infty}$ \\
\hline 11 & $9.63 \mathrm{e}-005$ & $9.63 \mathrm{e}-005$ & $9.65 \mathrm{e}-005$ & $9.68 \mathrm{e}-005$ \\
21 & $1.42 \mathrm{e}-005$ & $1.42 \mathrm{e}-005$ & $1.42 \mathrm{e}-005$ & $1.42 \mathrm{e}-005$ \\
41 & $2.99 \mathrm{e}-005$ & $2.99 \mathrm{e}-005$ & $3.00 \mathrm{e}-005$ & $3.00 \mathrm{e}-005$ \\
51 & $3.81 \mathrm{e}-005$ & $3.81 \mathrm{e}-005$ & $3.82 \mathrm{e}-005$ & $3.83 \mathrm{e}-005$ \\
71 & $5.48 \mathrm{e}-005$ & $5.48 \mathrm{e}-005$ & $5.48 \mathrm{e}-005$ & $5.50 \mathrm{e}-005$ \\
91 & $7.43 \mathrm{e}-005$ & $7.43 \mathrm{e}-005$ & $7.44 \mathrm{e}-005$ & $7.46 \mathrm{e}-005$ \\
\hline$N=50, M=70$ & & & & \\
\hline $\mathrm{n}$ & & & & \\
\hline 4 & $3.03 \mathrm{e}-004$ & $3.03 \mathrm{e}-004$ & $3.04 \mathrm{e}-004$ & $3.05 \mathrm{e}-004$ \\
5 & $4.10 \mathrm{e}-005$ & $4.10 \mathrm{e}-005$ & $4.11 \mathrm{e}-005$ & $4.12 \mathrm{e}-005$ \\
7 & $7.87 \mathrm{e}-005$ & $7.86 \mathrm{e}-005$ & $7.88 \mathrm{e}-005$ & $7.90 \mathrm{e}-005$ \\
9 & $1.07 \mathrm{e}-004$ & $1.07 \mathrm{e}-004$ & $1.07 \mathrm{e}-004$ & $1.07 \mathrm{e}-004$ \\
11 & $1.67 \mathrm{e}-004$ & $1.67 \mathrm{e}-004$ & $1.68 \mathrm{e}-004$ & $1.68 \mathrm{e}-004$ \\
15 & $2.06 \mathrm{e}-004$ & $2.06 \mathrm{e}-004$ & $2.06 \mathrm{e}-004$ & $2.07 \mathrm{e}-004$ \\
\hline
\end{tabular}

Example 3. Finally we consider the following two dimensional initial-boundary value partial integro-differential equation

$$
\frac{\partial v}{\partial t}(x, y, t)-\int_{0}^{t} \beta_{1}(t-z) \Delta v(x, y, s) d z=f(x, y, t), x, y \in \Omega, t \geq 0,
$$

where $\beta_{1}(t)=(\pi t)^{\frac{-1}{2}}$, and initial and boundary conditions are chosen as

$$
v(x, y, 0)=0, x, y \in \Omega, v(x, y, t)=v(x, y, t)=0, x, y \in \partial \Omega, t \geq 0,
$$


TABLE 4. Approximate solution corresponding to Example 3.

\begin{tabular}{ccccc}
\hline$N=50, n=5$ & $\alpha=0.1$ & $\alpha=0.25$ & $\alpha=0.6$ & $\alpha=0.75$ \\
\hline $\mathrm{M}$ & $l_{\infty}$ & $l_{\infty}$ & $l_{\infty}$ & $l_{\infty}$ \\
\hline 20 & 0.0112 & 0.0112 & 0.01 & 0.0112 \\
30 & 0.0019 & 0.0019 & 0.01 & 0.0019 \\
45 & $1.99 \mathrm{e}-004$ & $1.99 \mathrm{e}-004$ & $1.99 \mathrm{e}-004$ & $1.99 \mathrm{e}-004$ \\
60 & $1.46 \mathrm{e}-005$ & $1.46 \mathrm{e}-005$ & $1.46 \mathrm{e}-005$ & $1.47 \mathrm{e}-005$ \\
70 & $4.10 \mathrm{e}-005$ & $4.10 \mathrm{e}-005$ & $4.11 \mathrm{e}-005$ & $4.12 \mathrm{e}-005$ \\
90 & $3.70 \mathrm{e}-005$ & $3.69 \mathrm{e}-005$ & $3.70 \mathrm{e}-005$ & $3.71 \mathrm{e}-005$ \\
\hline
\end{tabular}

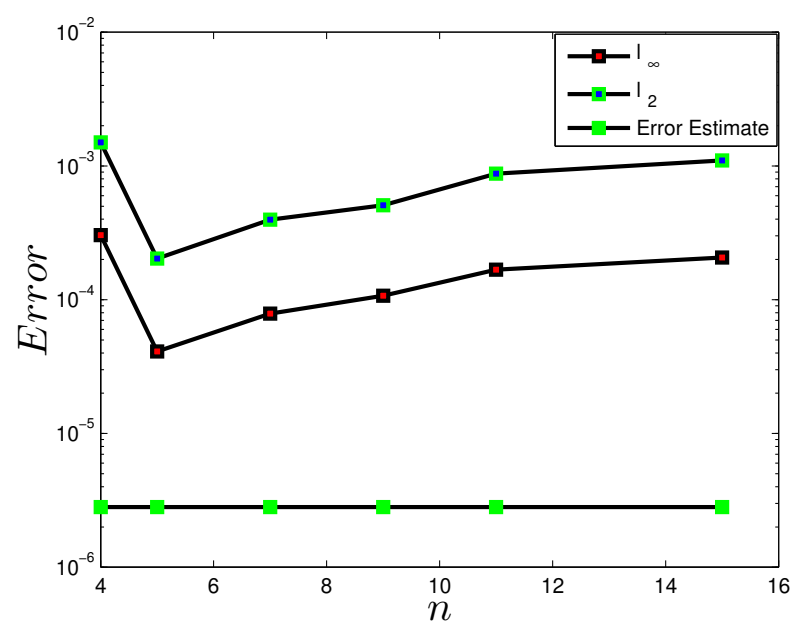

FIGURE 3. Approximate solution: error versus quadrature points corresponding to Example 3

where the exact solution $v(x, y, t)=t^{\frac{3}{2}}\left(1-x^{2}\right)\left(1-y^{2}\right)$ of this problem is selected from [34]. The parabolic contour $z=v(i w+1)^{2}$ for the strip $w=\eta+i c$, where $-\infty<\eta<\infty$ which reduces to

$$
z(\eta)=v\left((1-c)^{2}-\eta^{2}\right)+2 i v \eta(1-c),
$$

For the optimal values of the parameters corresponding to this path we used [40]. This problem is approximated over the domain $[-1,1]^{2}$. The error estimate $\varepsilon(c, M)$, $l_{\infty}$ error norm and $l_{2}$ error norm are displayed in Table 5. For various points $m$, $M=30$ quadrature points, $n=20$ stencil points the $l_{\infty}$ error norm and $l_{2}$ error norm decreasing steadily for $20 \leq M \leq 60$, while error estimate $\varepsilon(c, M)=e^{\frac{-c M}{\ln (M)}}$ for $c=1$ is decaying algebraically. The results are also computed for fixed collocation points $m=50$, quadrature pints $M=30$ and various stencil points $n$ in $\Omega_{i}$. The actual and 
numerical results are also displayed in Figure 4. Hence our numerical scheme is highly convergent for approximation of multi-dimensional partial integro-differential equations.

TABLE 5. Numerical results using Laplace transform based local RBF method corresponding to Example 4.

\begin{tabular}{ccccc}
\hline$(M, n)=(30,20)$ & \multicolumn{5}{c}{} \\
\hline$m$ & $l_{\infty}$ & $l_{2}$ & $\varepsilon(c, M)$ & RMS \\
\hline 20 & $1.77 \mathrm{e}-004$ & 0.0016 & 0.0013 & $7.92 \mathrm{e}-005$ \\
30 & $1.71 \mathrm{e}-004$ & $9.08 \mathrm{e}-004$ & $1.47 \mathrm{e}-004$ & $3.02 \mathrm{e}-005$ \\
40 & $1.31 \mathrm{e}-004$ & $8.45 \mathrm{e}-004$ & $1.95 \mathrm{e}-005$ & $2.11 \mathrm{e}-005$ \\
50 & $5.01 \mathrm{e}-005$ & $7.51 \mathrm{e}-004$ & $2.81 \mathrm{e}-006$ & $1.50 \mathrm{e}-005$ \\
60 & $6.72 \mathrm{e}-005$ & $6.97 \mathrm{e}-004$ & $4.32 \mathrm{e}-007$ & $1.16 \mathrm{e}-005$ \\
\hline$(M, m)=(30,50)$ & \multicolumn{5}{c}{} \\
\hline$n$ & $5.41 \mathrm{e}-004$ & 0.0616 & $2.81 \mathrm{e}-006$ & 0.0586 \\
\hline 10 & $5.01 \mathrm{e}-004$ & $7.51 \mathrm{e}-004$ & $2.81 \mathrm{e}-006$ & $1.50 \mathrm{e}-005$ \\
20 & $1.07 \mathrm{e}-004$ & 0.0016 & $2.81 \mathrm{e}-006$ & $3.13 \mathrm{e}-005$ \\
25 & $6.39 \mathrm{e}-005$ & 0.0015 & $2.81 \mathrm{e}-006$ & $3.01 \mathrm{e}-005$ \\
30 & $4.84 \mathrm{e}-005$ & 0.0015 & $2.81 \mathrm{e}-006$ & $2.97 \mathrm{e}-005$ \\
40 & $l_{2}=1.679 e-004[34]$ & & & \\
\hline \multicolumn{5}{c}{}
\end{tabular}

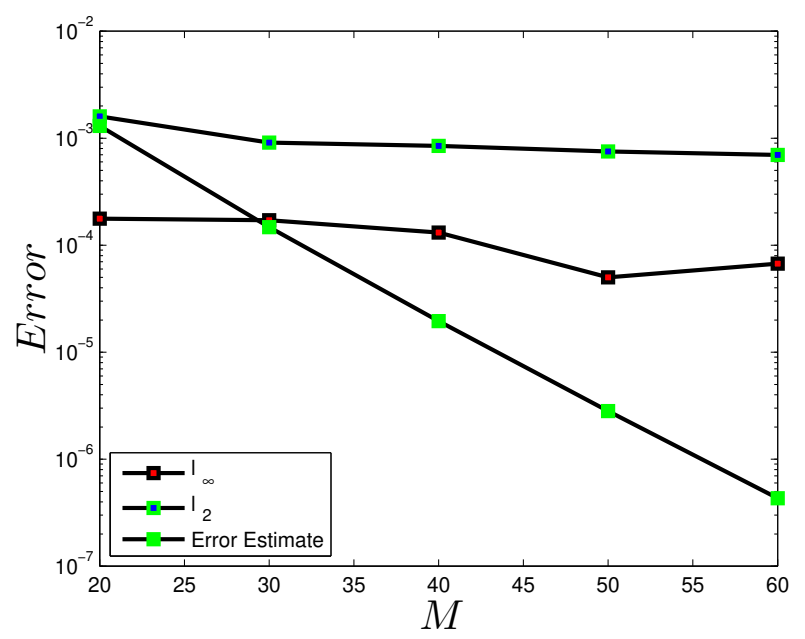

FIGURE 4. Numerical results corresponding to Example 4. 


\section{CONCLUSION}

In this work a numerical scheme is constructed which is based on Laplace transform and local radial basis functions method. The proposed numerical scheme efficiently approximated fractional order partial integro-differential equations with weakly singular kernel as well as memory term of convolution type. By application of Laplace transform time independent PDEs are obtained, after which the space variable is discretized using local meshless method. Finally the solution is obtained with inverse Laplace transform using quadrature rule along a contour with high accuracy. The accuracy and efficiency is of the numerical scheme is demonstrated and the present numerical method is an alternative for such type similar problems.

\section{REFERENCES}

[1] R. L. Bagley and P. Torvik, "A theoretical basis for the application of fractional calculus to viscoelasticity," Journal of Rheology, vol. 27, no. 3, pp. 201-210, 1983, doi: 10.1122/1.549724.

[2] H. Brunner, "Nonpolynomial spline collocation for Volterra equations with weakly singular kernels," SIAM journal on numerical analysis, vol. 20, no. 6, pp. 1106-1119, 1983, doi: $10.1137 / 0720080$.

[3] H. Brunner, "The approximate solution of Volterra equations with nonsmooth solutions," Utilitas Math, vol. 27, pp. 57-95, 1985.

[4] C. Chen, V. Thomée, and L. B. Wahlbin, "Finite element approximation of a parabolic integrodifferential equation with a weakly singular kernel," Mathematics of computation, vol. 58, no. 198, pp. 587-602, 1992, doi: 10.1090/S0025-5718-1992-1122059-2.

[5] A. M. Cohen, Numerical methods for Laplace transform inversion. Springer Science \& Business Media, 2007, vol. 5.

[6] X. Da, "On the discretization in time for a parabolic integrodifferential equation with a weakly singular kernel i: smooth initial data," Applied mathematics and computation, vol. 57, no. 1, pp. 1-27, 1993, doi: 10.1016/0096-3003(93)90010-C.

[7] T. Diogo, S. McKee, and T. Tang, "Collocation methods for second-kind Volterra integral equations with weakly singular kernels," Proceedings of the Royal Society of Edinburgh Section A: Mathematics, vol. 124, no. 2, pp. 199-210, 1994, doi: 10.1017/S0308210500028432.

[8] M. El-Shahed, M. Gaber, and M. Al-Yami, "The fractional q-differential transformation and its application," Communications in Nonlinear Science and Numerical Simulation, vol. 18, no. 1, pp. 42-55, 2013, doi: 10.1016/j.cnsns.2012.06.016.

[9] A. A. Elbeleze, A. Kılıçman, and B. M. Taib, "Approximate solution of integro-differential equation of fractional (arbitrary) order," Journal of King Saud University-Science, vol. 28, no. 1, pp. 61-68, 2016, doi: 10.1016/j.jksus.2015.04.006.

[10] G. E. Fasshauer, Meshfree approximation methods with MATLAB. World Scientific, 2007, vol. 6.

[11] S. Larsson, M. Racheva, and F. Saedpanah, "Discontinuous galerkin method for an integrodifferential equation modeling dynamic fractional order viscoelasticity," Computer Methods in Applied Mechanics and Engineering, vol. 283, pp. 196-209, 2015, doi: 10.1016/j.cma.2014.09.018.

[12] S. Larsson, V. Thomée, and L. Wahlbin, "Numerical solution of parabolic integro-differential equations by the discontinuous Galerkin method," Mathematics of Computation of the American Mathematical Society, vol. 67, no. 221, pp. 45-71, 1998, doi: 10.1090/S0025-5718-98-00883-7.

[13] W. Li and X. Da, "Finite central difference/finite element approximations for parabolic integrodifferential equations," Computing, vol. 90, no. 3-4, pp. 89-111, 2010, doi: 10.1007/s00607-0100105-0. 
[14] T. Lin, Y. Lin, M. Rao, and S. Zhang, "Petrov-Galerkin methods for linear Volterra integrodifferential equations," SIAM Journal on Numerical Analysis, vol. 38, no. 3, pp. 937-963, 2000, doi: 10.1137/S0036142999336145.

[15] M. López-Fernández and C. Palencia, "On the numerical inversion of the Laplace transform of certain holomorphic mappings,” Applied Numerical Mathematics, vol. 51, no. 2-3, pp. 289-303, 2004, doi: 10.1016/j.apnum.2004.06.015.

[16] W. McLean and V. Thomée, "Numerical solution of an evolution equation with a positivetype memory term," The ANZIAM Journal, vol. 35, no. 1, pp. 23-70, 1993, doi: $10.1017 / \mathrm{S} 0334270000007268$.

[17] W. McLean and K. Mustapha, "A second-order accurate numerical method for a fractional wave equation,” Numerische Mathematik, vol. 105, no. 3, pp. 481-510, 2007, doi: 10.1007/s00211-0060045-y.

[18] W. McLean, I. H. Sloan, and V. Thomée, "Time discretization via Laplace transformation of an integro-differential equation of parabolic type," Numerische Mathematik, vol. 102, no. 3, pp. 497522, 2006, doi: 10.1007/s00211-005-0657-7.

[19] W. McLean and V. Thomée, "Time discretization of an evolution equation via Laplace transforms," IMA journal of numerical analysis, vol. 24, no. 3, pp. 439-463, 2004, doi: 10.1093/imanum/24.3.439.

[20] W. McLean and V. Thomée, "Numerical solution via Laplace transforms of a fractional order evolution equation," The Journal of Integral Equations and Applications, pp. 57-94, 2010, doi: 10.1216/JIE-2010-22-1-57.

[21] W. McLean, V. Thomée, and L. B. Wahlbin, "Discretization with variable time steps of an evolution equation with a positive-type memory term," Journal of computational and applied mathematics, vol. 69, no. 1, pp. 49-69, 1996, doi: 10.1016/0377-0427(95)00025-9.

[22] K. S. Miller and B. Ross, An introduction to the fractional calculus and fractional differential equations. New York: John Wiley \& Sons, Inc., 1993.

[23] R. Miller, "An integrodifferential equation for rigid heat conductors with memory," Journal of Mathematical Analysis and Applications, vol. 66, no. 2, pp. 313-332, 1978, doi: 10.1016/0022247X(78)90234-2.

[24] A. Mohebbi, "Compact finite difference scheme for the solution of a time fractional partial integrodifferential equation with a weakly singular kernel," Mathematical Methods in the Applied Sciences, vol. 40, no. 18, pp. 7627-7639, 2017, doi: 10.1002/mma.4549.

[25] S. Momani and R. Qaralleh, "An efficient method for solving systems of fractional integrodifferential equations," Computers \& Mathematics with Applications, vol. 52, no. 3-4, pp. 459470, 2006, doi: 10.1016/j.camwa.2006.02.011.

[26] Y. Nawaz, "Variational iteration method and homotopy perturbation method for fourth-order fractional integro-differential equations," Computers \& Mathematics with Applications, vol. 61, no. 8, pp. 2330-2341, 2011, doi: 10.1016/j.camwa.2010.10.004.

[27] A. Pedas, E. Tamme, and M. Vikerpuur, "Spline collocation for fractional weakly singular integro-differential equations," Applied Numerical Mathematics, vol. 110, pp. 204-214, 2016, doi: 10.1016/j.apnum.2016.07.011.

[28] I. Podlubny, "Fractional differential equations, vol. 198 of mathematics in science and engineering," 1999.

[29] M. A. Ragusa, "Necessary and sufficient condition for a vmo function," Applied Mathematics and Computation, vol. 218, no. 24, pp. 11 952-11 958, 2012, doi: 10.1016/j.amc.2012.06.005.

[30] M. A. Ragusa and A. Tachikawa, "Boundary regularity of minimizers of $\mathrm{p}$ (x)-energy functionals," in Annales de l'Institut Henri Poincare (C) Non Linear Analysis, vol. 33, no. 2, doi: 10.1016/j.anihpc.2014.11.003, 2016, pp. 451-476. 
[31] A. Saadatmandi, M. Dehghan, and M.-R. Azizi, "The sinc-Legendre collocation method for a class of fractional convection-diffusion equations with variable coefficients," Communications in Nonlinear Science and Numerical Simulation, vol. 17, no. 11, pp. 4125-4136, 2012, doi: 10.1016/j.cnsns.2012.03.003.

[32] M. Saleh, S. Amer, M. Mohamed, and N. Abdelrhman, "Approximate solution of fractional integro-differential equation by Taylor expansion and Legendre wavelets methods," Cubo (Temuco), vol. 15, no. 3, pp. 89-104, 2013, doi: 10.4067/S0719-06462013000300009.

[33] K. Sayevand, M. Fardi, E. Moradi, and F. H. Boroujeni, "Convergence analysis of homotopy perturbation method for Volterra integro-differential equations of fractional order," Alexandria Engineering Journal, vol. 52, no. 4, pp. 807-812, 2013, doi: 10.1016/j.aej.2013.08.008.

[34] J. Tang and D. Xu, "The global behavior of finite difference-spatial spectral collocation methods for a partial integro-differential equation with a weakly singular kernel," Numerical Mathematics: Theory, Methods and Applications, vol. 6, no. 3, pp. 556-570, 2013, doi: 10.1017/S1004897900001033.

[35] T. Tang, "A finite difference scheme for partial integro-differential equations with a weakly singular kernel," Appl. Numer. Math, vol. 11, no. 4, pp. 309-319, 1993, doi: 10.1016/01689274(93)90012-G.

[36] M. Uddin, Z. Minullah, A. Ali et al., "On the local kernel based approximation of highly oscillatory integrals," Miskolc Mathematical Notes, vol. 16, no. 2, 2015, doi: 10.18514/MMN.2015.1091.

[37] M. Uddin, H. Ali, and A. Ali, "Kernel-based local meshless method for solving multi-dimensional wave equations in irregular domain," CMES-Computer Modeling in Engineering \& Scienses, vol. 107, no. 6, pp. 463-479, 2015.

[38] M. Uddin, Z. Minullah, and A. Ali, "On the approximation of rapidly oscillatory Hankel transform via radial kernels," Dolomites Research Notes on Approximation, vol. 11, no. 1, 2018.

[39] M. Uddin and M. Taufiq, "On the approximation of Volterra integral equations with highly oscillatory Bessel kernels via laplace transform and quadrature," Alexandria Engineering Journal, vol. 58, no. 1, pp. 413-417, 2019, doi: 10.1016/j.aej.2018.12.003.

[40] J. Weideman and L. Trefethen, "Parabolic and hyperbolic contours for computing the Bromwich integral," Mathematics of Computation, vol. 76, no. 259, pp. 1341-1356, 2007.

[41] S. Zhang, Y. Lin, and M. Rao, "Numerical solutions for second-kind Volterra integral equations by Galerkin methods," Applications of Mathematics, vol. 45, no. 1, pp. 19-39, 2000, doi: 10.1023/A:1022284616125.

[42] J. Zhao, J. Xiao, and N. J. Ford, "Collocation methods for fractional integro-differential equations with weakly singular kernels," Numerical Algorithms, vol. 65, no. 4, pp. 723-743, 2014, doi: 10.1007/s11075-013-9710-2.

Authors' addresses

\section{Marjan Uddin}

University of Engineering and Technology Peshawar Pakistan

E-mail address: marjan@uetpeshawar.edu.pk

Muhammad Taufiq

University of Engineering and Technology Peshawar Pakistan

E-mail address: taufiqduetpeshawar.edu.pk 